\title{
Cerebro-metacarpo-metatarsal Dystrophy (Pseudo-Pseudo Hypoparathyroidism) with Chromosomal Anomaly
}

\author{
J. JANCAR \\ From Stoke Park and Hortham/Brentry Hospital Groups, Bristol
}

\begin{abstract}
Albright, Burnett, Smith, and Parson (1942) investigated a female patient of 28 years who had suffered from idiopathic epilepsy since the age of 12 . Because the bones of the skull were unusually dense, hypoparathyroidism was suspected. The diagnosis was made when it was found that her Chwostek sign was positive and that her serum calcium and phosphorus levels were 6.4 and $6.0 \mathrm{mg}$. per $100 \mathrm{ml}$. respectively. Fortunately, it was decided, for certain academic reasons, to study the effect of parathyroid extract which is now no longer used in the routine treatment of hypoparathyroidism. She received $74 \mathrm{ml}$. (7,400 units) of parathyroid over a period of I2 days without any definite effect upon serum calcium or phosphorus levels. It was established that she had never received the parathyroid extract in the past, so that this amazing resistance to the hormone was not due to an antibody formation. Fortunately, she did respond well to dihydrotachysterol. She volunteered to undergo an exploration of the parathyroids, the biopsy of which showed normal parathyroid tissue. In addition, the authors described certain developmental abnormalities that served to delineate it from hypoparathyroidism, e.g. small stature, round face, and shortness of all fingers except the index finger, due to short metacarpals. They named the syndrome 'pseudo-hypoparathyroidism'.
\end{abstract}

Ten years later in 1952, Albright, Forbes, and Henneman reported a case of pseudo-hypoparathyroidism in which all the usual anatomical stigmata were present, but serum calcium and phosphorus levels were normal and the patient exhibited no clinical evidence of hypoparathyroidism. They called this new syndrome 'pseudo-pseudo hypoparathyroidism'.

Since these two original syndromes were described, the aetiology, relation, and nomenclature

Received November 4, 1963. have often been disputed. 'Pseudo-pseudo hypo- iv parathyroidism' was labelled by different authors $\vec{\omega}$ under the following names as collected by Cruz and $N$ Barnett (1962). (I) Alpha-hypocalcaemic pseudo- 은 hypoparathyroidism, and (2) alpha-hyperphos- phataemic-pseudo hypoparathyroidism (Albright et al., 1952); (3) dyschondroplasia and exostoses with metaphysial dysplasia or congenital skeletal malformations (Froom, 1956); (4) dyschondroplasia $\odot$ with soft tissue calcification and ossification and $c$ normal parathyroid functions (McNeely, Raisz, and LeMay, 1956); (5) dystrophie d'Albright Type II (Seringe and Tomkiewicz, I957); (6) brachymetacarpal dwarfism without tetany (Van der Werff ten Bosch, 1959); and (7) dyschondroplastic oligo- $\mathbb{D}$ phrenic dwarfism (Nagant de Deuxchaisnes, Isaac, Jacquet, and Hoet, 1960; and de Sèze, Kahn, O Freneaux, and Greslé, I96I).

We coined the term 'cerebro-metacarpo-metatarsal dystrophy'. By doing so we did not want to add more confusion to that already existing, but to give a more descriptive name to the condition and thus enable readers to form a mental picture of the 3 . main pathology of the syndrome.

\section{Components of the Syndrome}

Cruz and Barnett (1962) reviewed published $\frac{D}{2}$ papers of 49 cases of pseudo-pseudo hypoparathyroidism, and added 2 of their own. An analysis $\mathcal{N}$ of their review shows great variations of congenital $N$ anomalies from case to case, and variations in its $N$ degree and frequency. However, the following are $\frac{\omega}{\sigma}$ the most common features of the syndrome: (a) shortened metacarpal and/or metatarsal bones; (b) normal serum calcium and phosphorus; (c) short stature; (d) obese or stocky build; (e) broad :or round face; (f) mental retardation; and (g) 0 abnormal EEG record. In addition there is a marked preponderance of female to male patients $(4: I)$. 


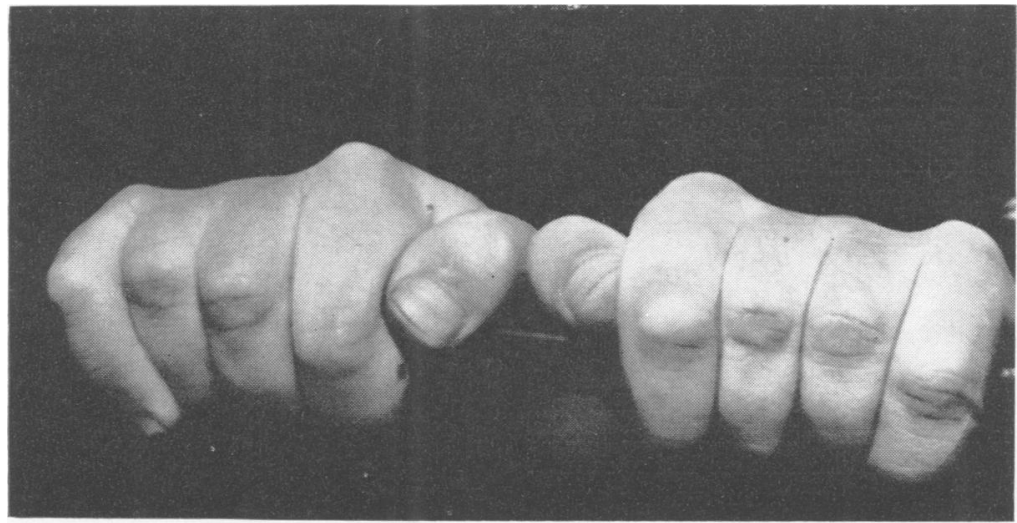

Fig. I. Patient's clenched fists showing concave metacarpo-phalangeal line.

Metacarpo-phalangeal Line of the Clenched Fist. When studying reported cases and investigating our case, we noticed abnormal metacarpophalangeal line, and we considered it to be a very useful diagnostic sign of the syndrome. Normally the line is slightly convex. In 'cerebro-metacarpo-metatarsal dystrophy' the line is concave (Fig. I), straight, or irregular, depending on shortening of the metatarsal bones and occurring in the following order of frequency, IV, V, III, I, II.

An abnormal line may also be found in other congenital abnormalities, but constant pathognomonic stigmata of those syndromes rule out 'cerebrometacarpo-metatarsal dystrophy'.

Other Physical Abnormalities. There are numerous other physical abnormalities that accompany the syndrome. We shall enumerate the more frequent ones in the sub-headings in accordance with the organs involved.

Skeletal Deformities: microcephaly, hyperostosis frontalis interna, calcification of basal ganglia, pectus excavatum, coxa vara, radius curvus, genu valgum, cubitus valgus, dysplastic hips or acetabula, bowing of the long bones; absence of, or presence of, extra phalanges or digits, syndactyly of the fingers or toes, dental hypoplasia, unerupted permanent teeth.

Glands: hyperthyroidism, hypothyroidism, diabetes mellitus, gonadal dysgenesis, menstrual irregularities, cystic ovaries.

Eye: cataracts, blue sclerae, and squints.

Ears: defective hearing, adherent ear lobules.

Others: epilepsy, psychiatric problems, hypertension, kidney disease, congenital heart disease, cor pulmonale, rheumatic arthritis, gout, phaeochromocytoma, haemangiomata, cystocele, urethrocele, hydrops of the gall-bladder, unexplained splenomegaly, spastic colon, atrophic vagina.

Aetiology. From the data available on the family tree of the $5 \mathrm{I}$ patients, the fact emerges that there is a history of physical or mental defect in the relatives, sometimes similar to those of the patients, and sometimes they only have some features of the syndrome.

Jesserer (1959) in his critical and constructive paper, quotes the workers who were able to reproduce similar malformations in animals, using noxious substances in early pregnancies. He also noted that the most critical period of development of the skeleton and parathyroid was between 6 and 8 embryonic weeks and that the most critical time for the development of the bones of the hands and feet was the seventh week. Facial bones, developing from the first visceral arch, are also most vulnerable during the seventh week of embryonic life (Jancar, I96I).

Further study of these vulnerable periods of embryonic development and the mechanism of malformation, either by heredity or by noxious substances, is necessary.

With the recent advances in the knowledge of chromosomes and further progress in biochemistry, the causes of this and other congenital abnormalities are nearer elucidation.

\section{Case Report}

A girl, the second of 3 children, all girls, was born at full-term on January 21,1936 , after a normal pregnancy and parturition, weighing $7 \mathrm{lb} .2 \mathrm{oz} .(3 \cdot 2 \mathrm{~kg}$.); the mother was 27 years and the father 25 years of age.

The child walked at 2 years and talked at 3 years. From the age of I I months she had had convulsions, and 
as a child she was always falling and constantly bruising herself.

Because of severe mental subnormality and epilepsy she attended a residential special school and training centre until she was admitted to Stoke Park Hospital for the mentally subnormal on August 15, 1962.

At 2 I years she developed psychiatric episodes and spent I month in a mental hospital for observation and treatment. Menses commenced at is years.

\section{Family History}

Physical Anomalies. Paternal grandmother, father, and younger sister have congenital absence of all upper molars. Father also had pulmonary tuberculosis. There is no evidence of other physical disorders in the family. The father was $5 \mathrm{ft} 3 \frac{3}{4}$ in. ( $162 \mathrm{~cm}$.); the mother $5 \mathrm{ft} 3 \frac{1}{4}$ in. ( $160.5 \mathrm{~cm}$.); elder daughter $5 \mathrm{ft} 3 \frac{1}{2} \mathrm{in}$. (I6I cm.); and younger daughter $5 \mathrm{ft} 2$ in. ( $157.5 \mathrm{~cm}$.).

Mental Illness. Father's uncle suffered from mental illness. The rest of the family are mentally normal.

Clinical Features. Height $5 \mathrm{ft} \mathrm{I}_{\frac{1}{4}}$ in. $(155.5 \mathrm{~cm}$.); weight 7 st. $5 \mathrm{lb}$. (50 kg.); and blood pressure $110 / 70$ mm.Hg.

The metacarpo-phalangeal line of the clenched fist is concave (Fig. I). She is of small stature with round face, has Harrison's sulcus, pectus excavatum, genu valgum, and suffers from slight kyphosis and lordosis. Low myopia is corrected by glasses. Neurological examination shows no abnormality.

She is mentally severely subnormal. On Terman Merrill testing-her mental age is 6.2 and I.Q. 4I. She is immature and emotionally unstable, and moody and anti-social at times. Her epilepsy is well controlled with phenobarbitone gr. I 3 times a day (60 mg. 3 times a day).

Investigations. The following investigations were carried out. Full blood count was normal; serum calcium, IO.I mg./100 ml.; serum inorganic phosphate (as P), $3.7 \mathrm{mg}$./100 ml.; serum cholesterol, I $5 \mathrm{I} \mathrm{mg./100}$ ml.; serum alkaline phosphatase, 7.3 K-A/100 ml.; electrolytes, normal; glucose tolerance test, normal; and Wassermann and Kahn reactions, negative.

Routine examination of urine was normal. Phenyl pyruvic, negative; total N, $0.6 \%$; cystine, normal; Sulkowitch calcium, normal; porphobilinogen, none detected.

Amino acid chromatogram showed normal 'glycinebeta-amino-iso-butyric' pattern.

A 24-hour specimen of urine showed calcium, 288 mg.; creatinine, I 34 g.; $\mathrm{N}$ methyl nicotinamide, normal; and 17 -ketosteroids, normal.

Cerebrospinal fluid was clear and colourless; cells and protein were normal. Calcium, $5 \mathrm{mg} . / 100 \mathrm{ml}$.; sugar, $55 \mathrm{mg}$./ $100 \mathrm{ml}$.; Lange: I I I I I I0000; Wassermann and Kahn, negative.

Radiography. Radiological examination of the chest revealed no abnormalities. The skull pictures showed a marked 'silver beaten' appearance of cranial vault, which

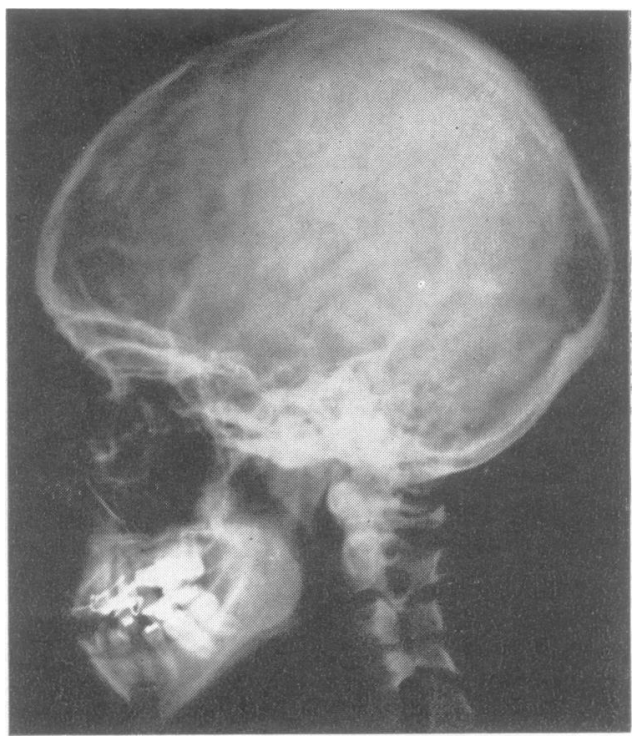

Fig. 2. Lateral view of skull radiograph, revealing marked 'silver $\vec{\theta}$ beaten' appearance.

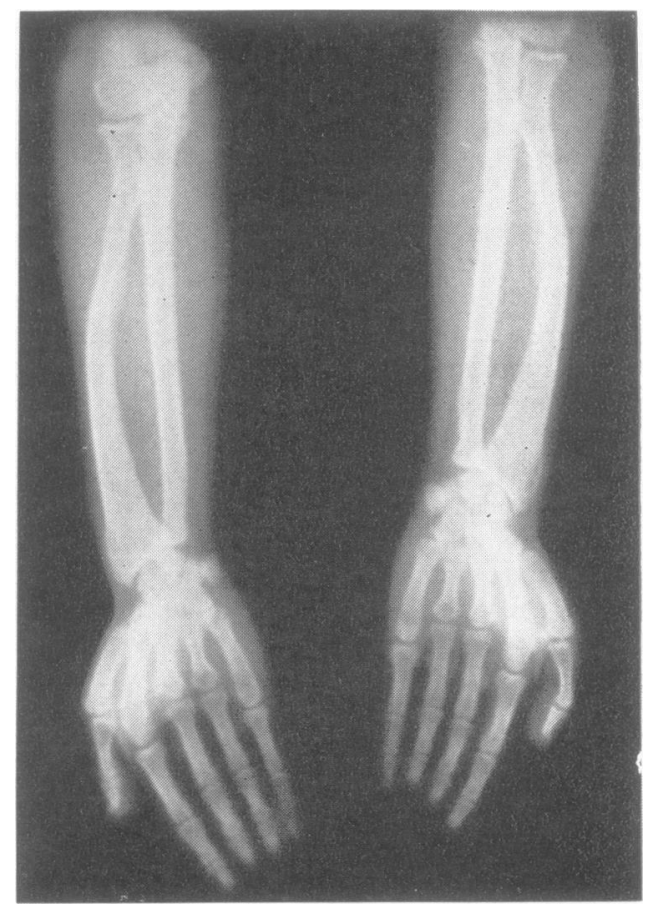

FIG. 3. Radiographs of the hands and forearms indicating shortening of the third and fourth metacarpals in both hands with some increase in the thickness of the second metacarpals; left and right radii are bowing and ulnae are slightly shorter 


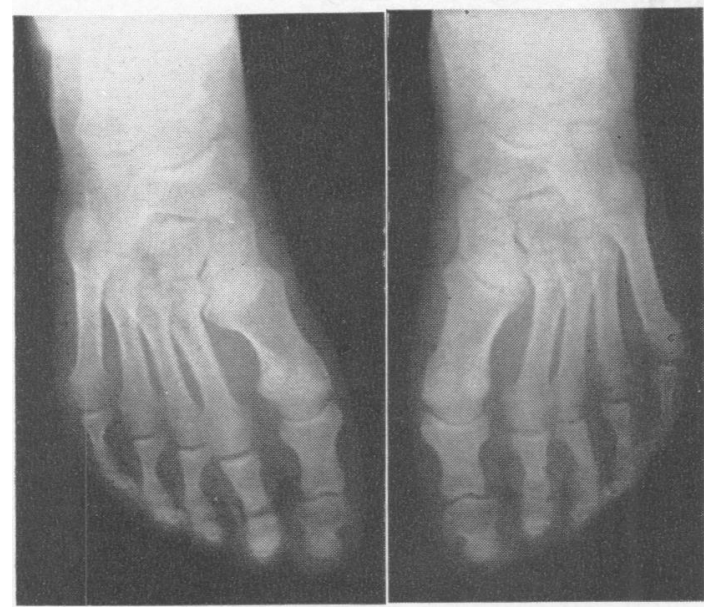

FIG. 4. Radiography of the feet. The metatarsals, especially the first one in the right foot, are a little shorter and thicker than normal.

is probably a persistence of the childhood anomaly. The sella is normal and this is very much against raised intracranial pressure (Fig. 2). Radiographs of the hands showed shortening of the third and fourth metacarpals in both hands with some increase in the thickness of the second metacarpals. The ulna bone is slightly shorter than normal with bowing of the radius on both sides (Fig. 3). The feet showed that the metatarsals, especially the first one in the right foot, were a little shorter and thicker than normal. No other lesion was seen (Fig. 4).

Air Encephalogram. The fourth ventricle was normally placed; the third ventricle was thin, and it was difficult to see the posterior margin clearly. Lateral ventricles showed some rounding of the upper angles, but their transverse diameters were within normal limits. The temporal horns were normally placed. Some cortical air was present and showed a little pooling in the frontal and parietal regions. There was evidence of a minimal atrophic lesion in the frontal lobes particularly, more so on the right side. The anatomy around the pineal gland was not seen. No other lesion was seen.

Electroencephalogram. A routine resting record was obtained followed by hyperpnoea and photic stimulation. The alpha rhythm was of low amplitude, partially responsive to opening the eyes and chiefly I I $\mathrm{c} / \mathrm{s}$. in frequency. This activity was more marked in the right hemisphere than the left. Bursts of low amplitude rhythmical theta activity were recorded from the right hemisphere and were most pronounced in the right temporal region. Hyperpnoea augmented the theta activity of the resting record and evoked theta components of a lower amplitude and high frequency in the left hemisphere. Photic stimulation augmented the right temporal theta activity and evoked a following response to a wide range of flash rates in the right hemisphere.
Cytological Investigation (Mrs Kusum P. Lele) The presence of satellites on all the acrocentric chromosomes has been generally agreed, although the maximum number of satellited chromosomes is seldom observed. The number of satellited chromosomes and the size of the satellites also varies between individuals. Tjio, Puck, and Robinson (1960) have reported unusually large satellites on one of the acrocentric chromosomes in two cases of Marfan's syndrome. Ellis and Penrose (I96I) have described enlarged satellites on one large acrocentric chromosome in three normal members of a family characterized by a number of abnormal pregnancies. As the karyotypes of the malformed members of this family could not be examined, the relation between the malformation and the enlarged satellites is not clear. However, the possibility that individuals possessing chromosomes with enlarged satellites are prone to produce offspring with malformations of the central nervous system cannot be excluded.

Cooper and Hirschhorn (I96I) reported 2 families with giant chromosomal satellites. In I family they were present on a small acrocentric and in the other on a large acrocentric. The abnormal propositi and the 3 normal members of both families possessed these giant satellites.

To study the chromosome pattern in the syndrome 'cerebro-metacarpo-metatarsal dystrophy', 2 cell lines of fibroblast cultures were set up from a skin biopsy, using the method described by Harnden (I960). Chromosome counts from 40 metaphases showed the chromosome number to be 46 . The karyotype was that of a normal female except for one large acrocentric chromosome, probably No. I5, which possessed unusually massive short arms with prominent satellites. The photographs of an analysed metaphase plate (Fig. 5) and six large acrocentrics from 5 different metaphases (Fig. 6) clearly illustrate this peculiar chromosome.

The chromosome complements in the parents' fibroblast cultures were normal and did not show the same anomalous large acrocentric chromosome.

The possibility of a causal relation between the massive short arms with prominent satellites on one large acrocentric chromosome and the syndrome cannot be excluded.

Dermatoglyphs (Prof. L. S. Penrose). Any disturbance of growth is liable to produce changes in the dermatoglyphic patterns on hands or feet. In the present case, the shortening of the third and fourth metacarpals did not produce any very noticeable effect of this kind. However, the position of the axial triradius, especially on the right hand, was more distal than usual (Fig. 7). In other respects the patterns appeared to be normal, though it might be possible to detect a characteristic tendency if prints of the hands of other cases with the same condition were obtained.

The left hallucal area showed a proximal tibial loop. This is a rare type of pattern that usually occurs in cases of trisomy of a large acrocentric. It is interesting to note that, in this patient, there was an excess of chromatin in one of these chromosomes.

Analysis of the fingertips is set out in the Table. 


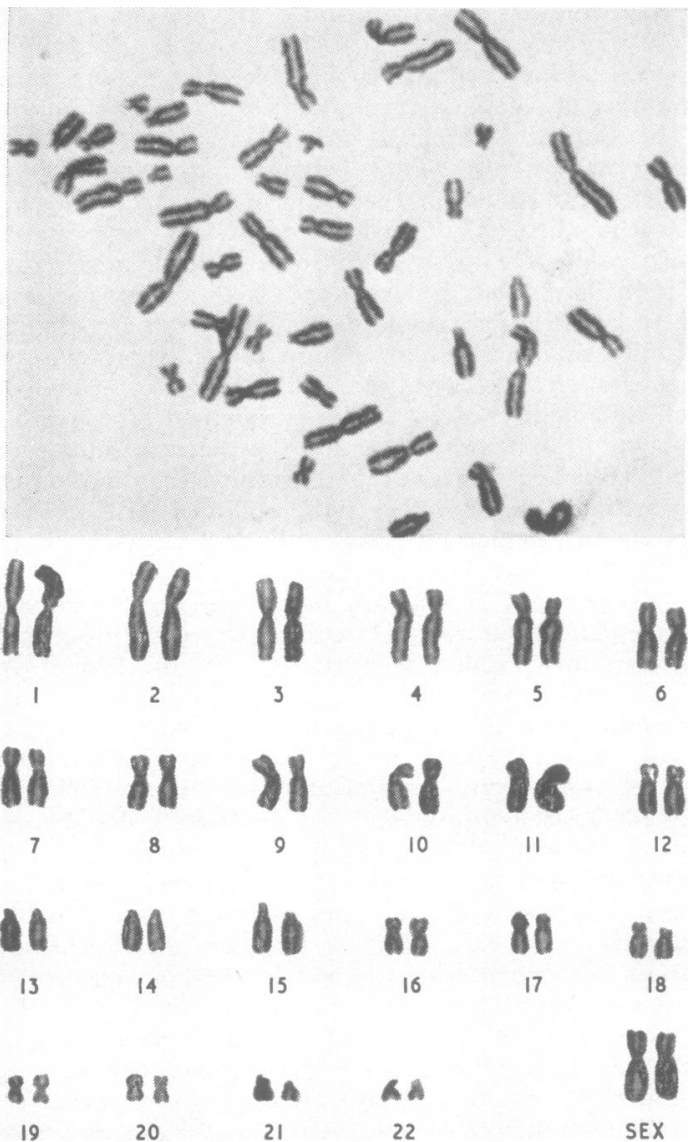

FIG. 5. Photograph of a dividing cell, with analysis.

TABLE

\begin{tabular}{|c|c|c|c|c|c|c|}
\hline \multirow[b]{2}{*}{ Hand } & \multicolumn{5}{|c|}{ Digits } & \multirow[b]{2}{*}{ Total } \\
\hline & $\mathbf{V}$ & IV & III & II & I & \\
\hline $\begin{array}{l}\text { Left } \\
\text { Right }\end{array}$ & $\begin{array}{l}\text { O/16 } \\
0 / 11\end{array}$ & $\begin{array}{l}7 / 21 \\
0 / 10\end{array}$ & $\begin{array}{l}0 / 15 \\
0 / 14\end{array}$ & $\begin{array}{l}12 / 12 \\
11 / 14\end{array}$ & $\left.\begin{array}{r}13 / 13 \\
0 / 17\end{array}\right\}$ & 143 \\
\hline
\end{tabular}

Note: $0 / 16$ indicates a radial count of 0 and an ulnar count of 16 ridges; $11 / 14$ indicates a radial count of $I I$ and an ulnar count of 14 ridges.

The total count is the sum of the figures in heavy type. When both radial and ulnar counts are zero, o/o, the pattern is an arch; when one count is zero the pattern is a loop, 16/0 shows an ulnar loop, o/16 shows a radial loop; when neither count is zero, $12 / 12$, the pattern is a whorl. The larger number for each finger is used for the total count.

The palms showed the following

\begin{tabular}{c|l|l|l}
\hline $\begin{array}{c}a-b \text { ridge count } \\
\text { Maximal atd } \\
\text { angle }\end{array}$ & $\begin{array}{l}\text { left } 4 \mathrm{I} \\
\text { left 48 }\end{array}$ & $\begin{array}{l}\text { right } 33 \\
\text { right 63 } 63^{\circ}\end{array}$ & $\begin{array}{l}\text { total } 74 \\
\text { total III }\end{array}$ \\
\hline
\end{tabular}

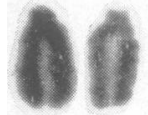

13
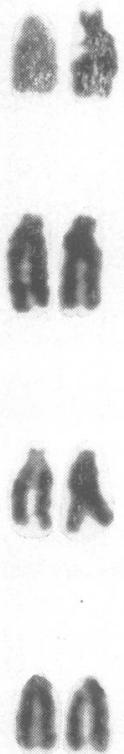

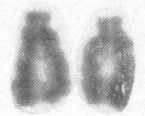

14

15
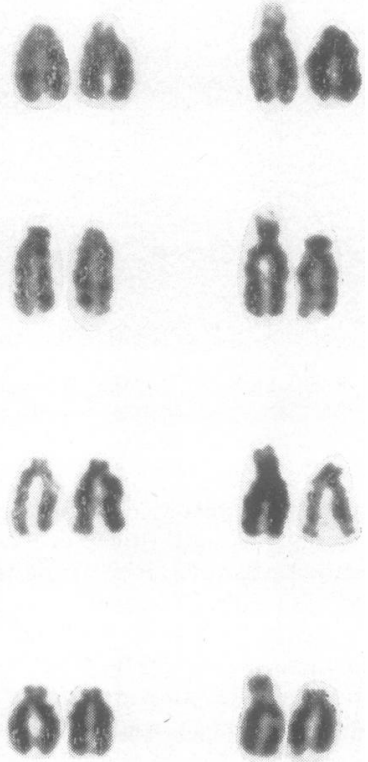

Fig. 6. Photographs of the 6 large acrocentric chromosomes from cells at metaphase.

\section{Summary}

The history and the evolution of the syndrome, pseudo-pseudo hypoparathyroidism, was discussed and renamed according to its main pathological changes as 'cerebro-metacarpo-metatarsal dystrophy'. The description of the syndrome, the aetiology, and the other associated physical abnormalities were noted

A pathognomic 'metacarpo-phalangeal line' of the clenched first was described.

A severely subnormal female patient suffering from 'cerebro-metacarpo-metatarsal dystrophy' was investigated, and confirmatory biochemical, radiological, and electrocardiographic findings were reported. The family history revealed that the $\mathrm{C}$ patient's paternal grandmother, her father, and her 2 younger sister suffered from congenital absence of $c$ all upper molars.

The study of the patient's chromosome pattern showed one large acrocentric chromosome, probably No. 15, which possessed unusually massive short arms with prominent satellites. The parents' chromosomes were normal. 

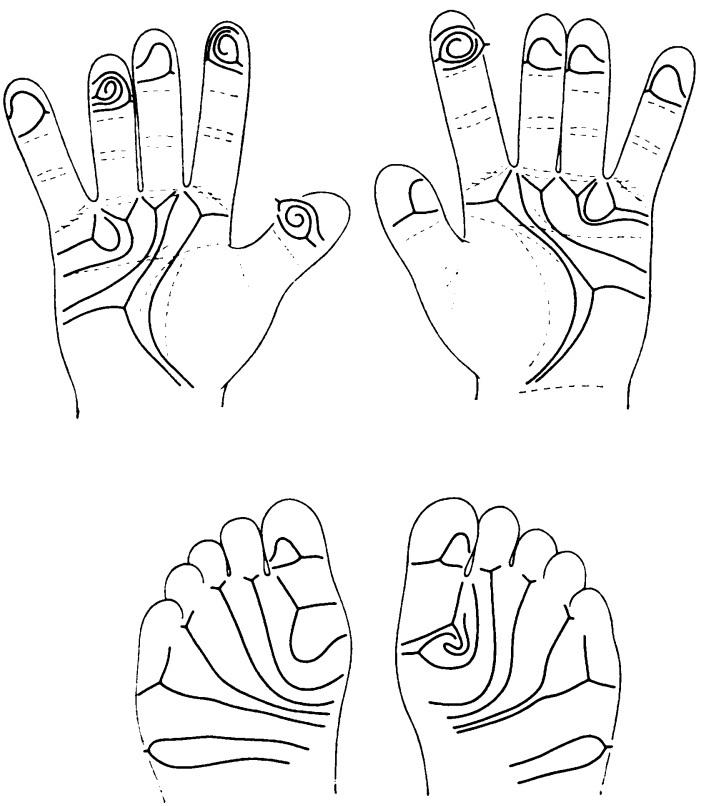

Fig. 7. Main lines of dermal ridge patterns on fingertips, palms, and soles.

The analysis of the dermatoglyphs of the case demonstrated that the position of the axial triradius, especially on the right hand, was more distal than usual and the left hallucal area showed a proximal tibial loop. This is a rare type of pattern which usually occurs in the cases of trisomy of a large acrocentric.

I wish to thank Professor L. S. Penrose, The Galton Laboratory, University College, London, for the helpful advice and the analysis of dermatoglyphs; Professor C. E. Dent, University College Hospital Medical School, London, for urine chromatography; Mrs Kusum P. Lele, M.Sc., Hon. Research Assistant, The Galton Laboratory, University College, London, for cytological investigations (the work was supported in part by a grant RG 6984 from the United States Public Health Service); Dr R. D. Eastham, Consultant Pathologist and his staff, Frenchay Hospital, Bristol, for the biochemical estimations; Dr W. Grey Walter, Burden
Neurological Institute, Bristol, for EEG interpretation; Mr J. J. Peters, for radiography and photography; and Miss J. E. Faithfull for secretarial work.

\section{REFERENCES}

Albright, F., Burnett, C. H., Smith, P. H., and Parson, W. (1942). Pseudo-hypoparathyroidism, an example of 'Seabright-Bantam' syndrome. Report of 3 cases. Endocrinology, 30, 922.

, Forbes, A. P., and Henneman, P. H. (I952). Pseudo-pseudohypoparathyroidism. Trans. Ass. Amer. Phycns, 65, 337.

Cooper, H. L., and Hirschhorn, K. (196I). Giant chromosomal satellites: an asymptomatic familial condition. Proc. 2nd Int. Conf. hum. Genet., Rome (Excerpta Med. Int. Cong. Ser., 32), p. E 102 (abstract).

Cruz, C. E., and Barnett, N. (1962). Mental retardation on pseudopseudohypoparathyroidism. Case reports of siblings and review of literature. Amer. F. ment. Defic., 67, $38 \mathrm{r}$.

Ellis, J. R., and Penrose, L. S. (196I). Enlarged satellites and multiple malformations in the same pedigree. Ann. hum. Genet., 25, 159.

Froom, G. A. (1956). Concerning the term 'pseudo-pseudohypoparathyroidism'. F. clin. Endocr., 16, 293.

Harnden, D. G. (1960). A human skin culture technique used for cytological examinations. Brit. F. exp. Path., 4I, 31 .

Jancar, J. (196I). Mandibulo-facial dysostosis (Berry-Franceschetti syndrome). F. Irish med. Ass., 48, 145.

Jesserer, H. (1959). Gibt es einen Pseudo-hypoparathyreoidismus? Eine kritische Analyse der bisherigen Erfahrungen. Klin. Wschr., 37, 394 .

McNeely, W. F., Raisz, L. G., and LeMay, M. (1956). Dyschondroplasia with soft tissue calcification and ossification and normal parathyroid function ('pseudo-pseudohypoparathyroidism'). Amer. F. Med., $21,649$.

Nagant de Deuxchaisnes, C., Isaac, G., Jacquet, A., and Hoet, J. J. (I960). Étude clinique et physio-pathogénique de la 'dystrophie d'Albright' (Pseudo-pseudo-hypoparathyroidisme) et de syndromes voisins, à propos de trois nouveaux cas, dont deux familiaux. Rev. franç. Étud. clin. biol., 5, 153.

Seringe, P., and Tomkiewicz, S. (I957). La dystrophie d'Albright pseudo-pseudo-hypoparathyroïdisme de cet auteur. Sem. Hôp. Paris, 33, 1092.

de Sèze, S., Kahn, M. F., Freneaux, B., and Greslé, C. (I96I). Un cas de dystrophie d'Albright 'pseudo-pseudo-hypoparathyroïdisme'. Rev. Rhum., 28, 123.

Tjio, J. H., Puck, T. T., and Robinson, A. (1960). The human chromosomal satellites in normal persons and in two patients with Marfan's syndrome. Proc. nat. Acad. Sci. (Wash.), 46, 532.

Van der Werff ten Bosch, J. J. (1959). The syndrome of brachymetacarpal dwarfism ('pseudo-pseudohypoparathyroidism') with and without gonadal dysgenesis. Lancet, $1,69$.

\section{Addendum}

Since this paper was submitted for publication, we have seen three more typical cases of cerebro-metacarpo-metatarsal dystrophy. They are three mentally defective sisters with I.Q.s of 34,35 , and 30 , and chronological ages of 42,34 , and 30 respectively. A full report on the cases will be published at a later date. 